\title{
Elementos de influencia en la toma de decisiones entre directores comerciales en empresas familiares. Estudio comparado de empresas de Cataluña (España) y Barranquilla (Colombia)
}

\section{Marcela Navia-Núñez, Yanna Stefanu-Lidorikiotu, Antonio Valdivia-Ubeda}

Infocaribe, Barranquilla, Colombia

Facultad de Economía y Empresa, Universidad de Barcelona

Facultad de Economía y Empresa, Universidad de Barcelona

El director comercial es el enlace entre la empresa y el mercado. Como parte de su función, toma muchas decisiones, proceso en el que pueden intervenir otras personas y se pueden dar diversas situaciones. El presente estudio analiza las influencias en el proceso de toma de decisiones de directores comerciales de empresas familiares. En una primera etapa, se realizaron entrevistas en profundidad en Cataluña (España) a directores comerciales de empresas de este tipo; seguidamente, el mismo instrumento se aplicó en Barranquilla (Colombia). Tras analizar sus decisiones bajo un enfoque sistémico, se construyeron diagramas de flujo, en los que se plasma el proceso de decisión. Se establecieron nueve subsistemas que fueron clasificados de la siguiente manera: ajeno a la empresa, entorno, empresa, familia-propiedad, órganos de dirección, información, otro personal de la empresa, subordinados y director comercial. A partir del análisis de las interrelaciones entre el director comercial y los demás subsistemas, se identificó que estos últimos pueden iniciar, promover, reforzar, ralentizar, modificar, parar o anular las decisiones de los directores comerciales. En ambas regiones, se detectaron fuertes influencias de los otros subsistemas en las decisiones de los directores comerciales, con pequeñas diferencias entre Cataluña y Barranquilla.

Palabras clave: Proceso de toma de decisiones, enfoque sistémico, empresa familiar y dirección comercial

\section{Influencing elements on the decision-making process of commercial directors in family enterprises: A com- parative study between organizations in Catalonia (Spain) and Barranquilla (Colombia)}

Commercial managers are links between their organizations and their markets. They are responsible for several decisions, in which many other people take part under different circumstances. This study analyzes the influencing elements in the decision-making processes of commercial managers who work in family enterprises. As a first step, the researchers held in-depth interviews to commercial directors of family enterprises in Catalonia (Spain). The same instrument was used then in Barranquilla (Colombia). As result of the analysis under a systemic approach, the authors established flow diagrams showing the commercial directors' decision processes. Nine subsystems emerged from the analysis: external to the enterprise, environment, enterprise, family-property, direction staff, other enterprise members, working team, and commercial director. Relationships among commercial directors and the other subsystems were determined showing that these subsystems may start, promote, reinforce, slow down, modify, stop or invalidate decisions of commercial directors. These influences from the other subsystems on decisions of commercial directors were found to be strong, with little differences, in both Catalonia and Barranquilla.

Keywords: Decision-making process, family property, systemic approach, commercial director 


\section{Elementos da influência na tomada de decisóes entre diretores comerciais em empresas familiares: Estudo comparativo das empresas na Catalunha (Espanha) e Barranquilha (Colômbia)}

O gerente comercial é o link entre a empresa e o mercado. Toma muitas decisōes; no processo podem intervir outras pessoas e podem apresentar diversas situações. O presente estudo faz uma revisão das influências no processo de tomada de decisōes dos diretores comerciais das empresas familiares. Em uma primeira etapa foram feitas entrevistas em profundidade na Catalunha (Espanha) para gerentes comerciais das empresas deste tipo; a seguir, o mesmo instrumento se aplicou em Barranquilha (Colômbia). Depois de analisar suas decisóes sob uma abordagem sistêmica, os fluxogramas foram estabelecidos onde o processo de decisão é capturado; foram estabelecidos nove subsistemas que foram classificados como: alheio ao empresa, entorno, empresa, família propriedade, órgãos de gestáo, informação, outro pessoal da empresa, subordinados e diretor comercial. As inter-relaçôes entre o gerente comercial e os demais subsistemas foram analisadas e encontraram-se que estes últimos podem iniciar, promover, reforçar, desacelerar, modificar, parar ou cancelar as decisóes dos diretores comerciais. Em ambas as regiōes se detectaram fortes influências dos outros subsistemas nas decisões dos diretores comerciais, com pequenas diferenças entre Catalunha e Barranquilha.

Palavras chave: Processo na toma de decisóes, abordagem sistêmica, empresa familiar e gerência comercial

\section{Introducción}

Uno de los temas que más inquietud causa en la administración de empresas es la toma de decisiones, que se convierte en una actividad cotidiana e irrenunciable en todos los niveles de la organización.

Herbert Simon (1947), el teórico más reconocido en el tema, define el proceso de toma de decisiones así: 'todo comportamiento implica una selección, consciente o inconsciente, de determinadas acciones, entre todas las que son físicamente posibles, para el que actúa y para las personas sobre las cuales este ejerce influencia o autoridad'; selección porque, al hacerla, se renuncia a otras líneas de acción. (citado en Navia Núñez, 2002a, p. 54)

En esta definición, se plantea otro tema de interés: las decisiones se toman bajo influencias de otras personas y situaciones. El presente artículo se centra en este aspecto en un escenario específico: el cargo de director comercial; la empresa de tipo familiar, donde las relaciones interpersonales adquieren una dinámica más allá del ámbito laboral; y en los contextos de Cataluña (España) y Barranquilla (Colombia), dado que la investigación compara los resultados encontrados en ambas regiones. También, se realiza una comparación entre el estudio actual llevado a cabo en Barranquilla y uno similar que tuvo lugar en la misma ciudad en 2002.

\section{Conceptos}

Uno de los teóricos que estudió el tema de la influencia personal fue Lazarsfeld. Su trabajo analizó cómo el «voz a voz» de los líderes de opinión puede hacer cambiar el voto de una persona, incluso, más que los medios de comunicación masiva. Los planteamientos de Lazarsfeld se pueden aplicar al ámbito de la toma de decisiones empresariales, pues los directivos escuchan conceptos de otras personas. Lazasferld, Berelson y Gaudet (1944) descubrieron que el prestigio de quien ejerce la influencia hace que pueda tener un papel más o menos relevante, y que algunas de las conversaciones que más influyen suceden con familiares y amigos. $\mathrm{Al}$ respecto, cabe anotar que el diccionario de la Real Academia Española define la influencia como «poder, valimiento, autoridad de alguien para con otra u otras personas o para intervenir en un negocio», así como 
«persona con poder o autoridad con cuya intervención se puede obtener una ventaja, favor o beneficio» (2001, p. 1274).

En otro sentido, uno de los campos de aplicación de la teoría de sistemas es el ámbito de las organizaciones empresariales, que se han interpretado como sistemas abiertos. Chiavenato afirma que «la palabra sistema denota un conjunto de elementos interdependientes e interactuantes o un grupo de unidades combinadas que forman un todo organizado» (2004, p. 411). Así, las organizaciones son seres vivos, que cambian y se adaptan a su entorno.

Como explica Aktouf (1998, p. 148) basándose en Taylor, la decisión es lo que sucede entre el pensamiento y la acción; la forma en que se llega a la opción es el proceso de decisión.

Este proceso implica una serie de actos parciales y secuenciales que conducirán al decisor desde la toma de consciencia de la necesidad de elegir, hasta seleccionar una solución entre las más adecuadas, tomando en cuenta la situación, y pasando por la recolección y el procesamiento de toda la información necesaria. (Aktouf, 1998, p. 148)

En el ámbito empresarial, hay incertidumbre y presión por tomar decisiones rápidamente. En ese marco, se terminan manejando supuestos y se busca «sustentar analíticamente por qué se cree que las condiciones históricas cambiarán o no y de qué manera esos cambios pueden afectar a la decisión» (Cachanosky, 2000, p. 5). Otros factores pueden incidir en la toma de decisiones, como la personalidad y la experiencia de quien la toma, la complejidad de la situación y las circunstancias.

Minztberg (1991), citado por Stefanu (2011), describe los papeles que desempeña el director comercial en la toma de decisiones: como empresario, busca mejorar la unidad; como gestor de anomalías, debe responder involuntariamente a las presiones; como asignador de recursos, trabaja por delegar decisiones a sus subordinados; y, como negociador, establece acuerdos ante otros departamentos o entidades fuera de la organización. Stefanu indica que no hay unanimidad entre los autores acerca de si la dirección comercial incluye la función de mercadeo. Artal (2005), por su parte, determina tres áreas de las decisiones a las que se enfrenta el director comercial: estratégicas, aquellas que se relacionan con estructura, clientes, demanda y territorios; gestionarias, que abarcan procesos de selección, formación, motivación y remuneración; y evaluativas, que refiere a los resultados, los comportamientos, los costes y la rentabilidad. Según Prado, Alberton y Lana, «los antecedentes teóricos indican que la estructura de la propiedad y el tipo de gestión pueden influir en el rendimiento de las empresas» (2016, p. 36), lo cual aplica al caso de las compañías familiares.

No es fácil encontrar una definición unificada de empresa familiar. Algunos autores señalan que es aquella en la que los miembros de una familia tienen votos, mientras que otros hacen alusión a que los familiares tienen cargos directivos. Asimismo, unos autores sostienen que la familia debe tener la aspiración de conservar la empresa de generación en generación, mientras que otros no prestan atención a ese aspecto. De este modo, se muestra que hay un abanico de posibilidades, precisamente, porque las empresas son sistemas en movimiento. Neubauer y Lank indican que una empresa familiar «es aquella empresa, sea unipersonal o sociedad mercantil de cualquier tipo, en la que el control de los votos está en manos de una familia determinada» (1999, p. 37). Para Sánchez-Crespo y Sánchez, «es cualquier empresa dirigida por miembros de una familia, que son sus propietarios» (2001, p. 29).

En Hispanoamérica, la empresa familiar cumple un rol importante: en América Latina, 9 de cada 10 empresas 
son familiares; y en España, el 71\% (Belausteguigoitia, 2005 y Gómez Betancourt, 2006, citados en González, Castruita y Mendoza, 2018).

\section{Método}

En su tesis doctoral para la Universidad de Cádiz, Stefanu (2011) realizó una investigación cualitativa con la técnica de entrevista en profundidad, aplicada a 43 directores comerciales de empresas familiares en Cataluña, España. En este estudio, se entendió «director comercial» como el responsable de la planificación, organización y control del equipo de ventas (Santesmases, 1999, citado en Stefanu 2011). En las entrevistas, se solicitó al entrevistado que recordara dos decisiones para analizar: una que hubiera tomado en su puesto de trabajo como director comercial y otra sobrevenida, es decir, una decisión tomada por iniciativa de una persona de otro cargo o que había sido tomada por otra persona y el entrevistado debía gestionarla. A partir de su descripción, se indagó en el proceso de la toma de decisión, las personas implicadas y los elementos que influyeron en esa toma de decisión, que es lo que permitió establecer el flujo de las influencias. Al final, se analizaron poco más de dos decisiones por entrevistado; la cifra final ascendió a un total de 92. La misma guía o pauta de entrevista se aplicó en Barranquilla, Colombia, en el segundo semestre de 2016 a 8 directores comerciales de empresas familiares. En cada caso, se analizaron los mismos dos tipos de decisión para un total de dieciséis decisiones. En el presente artículo, se realiza un análisis de los resultados de cada país y un análisis comparativo.

\section{Perfil de los entrevistados en Cataluńa}

Entre las 43 empresas, se seleccionaron diferentes sectores económicos como construcción, comercial (seguros, productos de limpieza, suministros médicos, automóviles), salud ambiental, servicios (jurídicos, transporte público, mantenimiento de maquinaria industrial), alimentos, metalúrgico o químico, entre otros. Hay empresas con gran cantidad de empleados, como una que cuenta con 600, de los cuales 100 pertenecen al área comercial, todos con contrato laboral. Hay otra del sector de la construcción con 1300 empleados, de los cuales 50 son ingenieros de ventas con contrato laboral. También, se incluyó empresas con menos de quince empleados, como una que distribuye electrodomésticos de línea blanca que cuenta con doce empleados, tres de los cuales son del área comercial, más un agente con contrato mercantil. Se tomó otra empresa de veinticinco empleados que presta servicios industriales; en el área comercial, hay ocho vendedores con contrato mercantil y seis con contrato laboral. Algunas de las empresas se mueven en mercados fuera de Espańa y varias atienden únicamente el mercado local o regional dentro del país.

Una empresa dedicada a los seguros en España está en la quinta generación de la familia. Tiene 45 empleados, todos del área comercial. El padre ocupa la posición de presidente, uno de los hijos es el director en Barcelona -quien fue el entrevistado-y el otro es el director financiero. El directivo entrevistado, quien inició su vida laboral en la empresa familiar, está empezando a asumir la dirección nacional como parte del relevo generacional. También, se encontró una empresa de 400 empleados que se fundó hace más de 100 años y está en la cuarta generación. La empresa fabrica alimentos y cosméticos, y tienen sus propios puntos de venta. Cuenta con 100 agentes externos. El entrevistado es miembro de la familia.

En otros casos, el entrevistado es un director comercial para Cataluña, como una empresa constructora. En una empresa multinacional con 12 empleados, se entrevistó al fundador del negocio que regenta los 
puestos de dirección general y dirección comercial de forma simultánea. También, se entrevistó a personas que no pertenecen a la familia, como en el caso de una empresa de 65 empleados que fabrica alimentos. El director comercial de esta institución es una de las pocas personas que domina varios idiomas; por ello, tiene un papel fundamental en mercados externos. Cabe anotar que varios de los entrevistados cuentan con estudios de maestría.

\section{Análisis de resultados en Cataluńa}

De acuerdo con lo encontrado en España (Stefanu, 2011), se clasificaron las influencias en nueve subsistemas: Ss1. Ajeno a la empresa, Ss2. Entorno, Ss3. Empresa, Ss4. Familia-propiedad, Ss5. Órganos de dirección, Ss6. Información, Ss7. Otro personal de la empresa, Ss8. Subordinados y Ss9. Director comercial. Cada subsistema, tiene varias fuentes de influencia. Por ejemplo, el Ss1 (ajeno a la empresa) aglutina 4 fuentes distintas: a) curso de formación, b) contactos personales del director comercial, c) contactos personales del director general y d) amigos del decisor. El Ss5 (órganos de dirección) reúne seis fuentes: a) modelo de dirección de la familia, b) modelo de dirección de dirección general, c) creencias y valores del comité de dirección, d) creencias y valores de dirección general, e) posición jerárquica de dirección general, y f) roles y estatus de dirección general en el mercado.

Se han detectado siete tipos de influencias: inicia, promueve (que la decisión siga adelante), refuerza (ejerce presión positiva), ralentiza (ejerce presión para que la decisión se retrase), modifica (ejerce presión para intentar modificar la decisión), para (durante un tiempo) y anula (los elementos de influencia provocan que finalmente la decisión no se llegue a consolidar). Los códigos se pueden apreciar en la Figura 1.
Figura 1. Códigos para los tipos de influencia

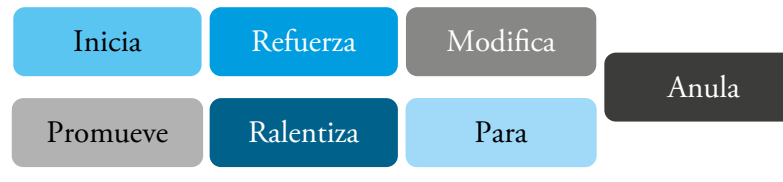

Con este modelo, se crearon diagramas de flujo con cuatro tipos de código:

- Subsistemas y sus fuentes de influencia con código alfanumérico (ya mencionado)

- El tipo de influencia que ejerce cada fuente, que figura en colores

- Las influencias entre subsistemas, señalados con flechas curvas (punteadas si la influencia afecta relativamente a la decisión y continua si la afecta plenamente)

- Relaciones entre las fuentes de influencia, marcados con flechas rectas punteadas

En la Figura 2, aparece el análisis de una decisión con pocas relaciones, mientras que, en la Figura 3 , se muestra un caso en que hubo muchas relaciones. «E» indica el número de empresa y «D» el número de decisión. «DI» refiere a la decisión inicial y «DF», a la decisión final. Las letras en minúscula indican la fuente de influencia concreta dentro de cada subsistema.

En la Figura 2, se aprecian los tres subsistemas que actúan en esta decisión: el entorno, los órganos de dirección y la información. Existen dos interrelaciones y, del mismo modo, hay dos influencias entre subsistemas, ambas fuertes. En cuanto a las fuentes de influencia, una ralentiza la decisión y otra la promueve, además del iniciador. En esta decisión, los tres hermanos que forman parte de la sociedad querían implementar elementos de seguimiento y control de la cartera de clientes por considerar poco rentable la forma actual. 
En la Figura 3, se observa que son cuatro los subsistemas implicados en la decisión: el ajeno a la empresa, el entorno, los órganos de dirección y el director comercial. El número de interrelaciones es de cuatro. Las influencias entre subsistemas son tres relativas y una clara. Dos influencias promueven la decisión, dos la refuerzan y una la inicia. En este caso el director comercial es quien inicia e involucra al director general para que su presencia en una visita facilite el acceso a los clientes, dado que él es un personaje conocido.

Teniendo en cuenta todas las decisiones analizadas de las empresas de Cataluña, Stefanu (2011) encontró que los subsistemas más activos en la toma de decisión del director comercial —además de él mismo— son el entorno, la familia-propiedad y los órganos de dirección.
El número de interrelaciones van desde dos hasta diez, pero lo más habitual es que se den tres interrelaciones. En cuanto a las influencias entre sistemas, la media es de tres por decisión tomada: dos de ellas son claras y una es relativa. La mayoría de las influencias promueven la decisión y pertenecen al subsistema del entorno. Por su parte, casi todas las influencias que ralentizan una decisión provienen del director comercial. Los intentos de modificar una decisión pueden tener su origen, principalmente, en el director comercial, en la familiapropiedad o en los órganos de dirección. Por su parte, los factores por los cuales se intentan parar las decisiones son, sobre todo, la familia-propiedad, los órganos de dirección u otro personal de la empresa. Las dos influencias anuladoras que se encontraron provienen una del entorno y la otra de los órganos de dirección.

\section{Figura 2: Representación de la decisión modificar la gestión de cartera de clientes}

\section{E8 - D20 - Modificar la gestión de la cartera de clientes}

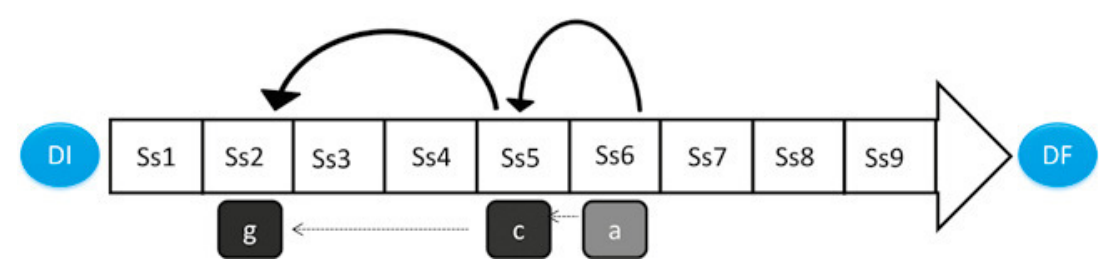

Figura 3. Representación de la decisión captación de un nuevo proyecto

E6 - D18 - Captación de un nuevo proyecto

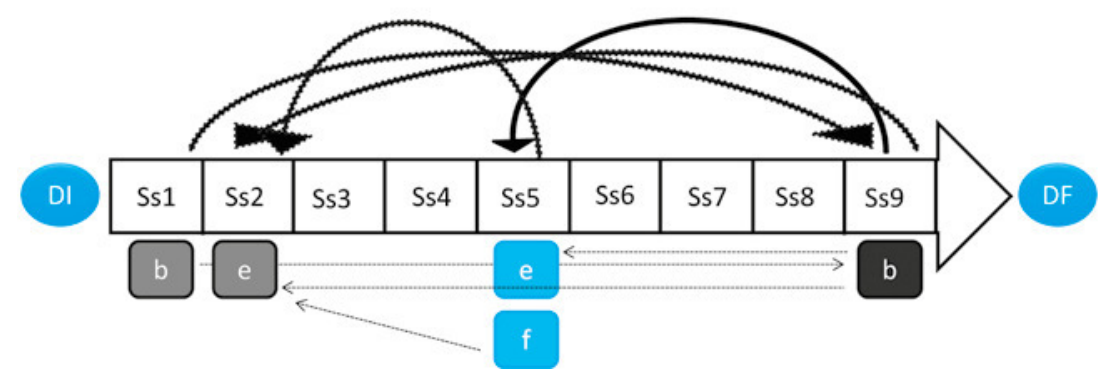




\section{Perfil de los entrevistados en Barranquilla}

El perfil de los entrevistados para el caso de Barranquilla, Colombia, fue el que se ilustra en la siguiente tabla.

\section{Tabla 1. Características de los directores comerciales entrevistados en Barranquilla}

\begin{tabular}{|c|c|}
\hline Criterio & Descripción \\
\hline $\begin{array}{l}\text { Es miembro } \\
\text { de la familia }\end{array}$ & $\begin{array}{l}\text { Cinco de los ocho entrevistados eran miembros } \\
\text { de la familia. Tres de ellos son de la segunda } \\
\text { generación y menores de } 35 \text { ańos. Los otros dos } \\
\text { de los cinco que son miembros de la familia son } \\
\text { fundadores: uno con dos hermanos, y otro tra- } \\
\text { baja con su hijo y su esposa. }\end{array}$ \\
\hline $\begin{array}{l}\text { No es } \\
\text { miembro de } \\
\text { la familia }\end{array}$ & $\begin{array}{l}\text { Una de las empresas surgió de la fusión de dos } \\
\text { empresas familiares; al fusionarse, las dos fami- } \\
\text { lias tomaron la decisión de no ocupar cargos y, } \\
\text { únicamente, formar parte de la junta directiva. }\end{array}$ \\
\hline Cargo & $\begin{array}{l}\text { Seis tienen el cargo de director comercial para } \\
\text { toda la empresa, una región o una unidad de } \\
\text { negocio. Dos son gerentes generales (y fundado- } \\
\text { res) que llevan la gerencia comercial. }\end{array}$ \\
\hline $\begin{array}{l}\text { Tamaño de } \\
\text { la empresa }\end{array}$ & $\begin{array}{l}\text { Según clasificación colombiana por activos, una } \\
\text { de las compañías es una microempresa, otra es } \\
\text { pequeña, cuatro son medianas y dos son grandes. }\end{array}$ \\
\hline $\begin{array}{l}\text { Sector eco- } \\
\text { nómico }\end{array}$ & $\begin{array}{l}\text { Hay seis empresas comerciales; cinco de ellas, } \\
\text { además, tienen un fuerte componente en servi- } \\
\text { cios (instalación de software, ensamble de artícu- } \\
\text { los para carga, mantenimiento de filtros, asesoría } \\
\text { en costos logísticos y corte de piezas en acero). La } \\
\text { otra es netamente comercial: solo distribuye; no } \\
\text { ofrece servicios adicionales ni fabrica. } \\
\text { Hay dos fábricas (de alimentos e industria quí- } \\
\text { mica). }\end{array}$ \\
\hline $\begin{array}{l}\text { Tiempo de } \\
\text { creación }\end{array}$ & $\begin{array}{l}\text { La empresa más joven tiene ocho años de exis- } \\
\text { tencia. Las más antiguas cuentan con más de } 50 \\
\text { años. }\end{array}$ \\
\hline $\begin{array}{l}\text { Tipo de } \\
\text { contratación } \\
\text { en el área } \\
\text { comercial }\end{array}$ & $\begin{array}{l}\text { La microempresa tiene al gerente general en } \\
\text { el área comercial y dos personas que no son } \\
\text { empleadas y ganan comisiones. } \\
\text { La figura de comisiones aparece en las dos } \\
\text { empresas grandes también, pero solo para casos } \\
\text { específicos: una para mercados exteriores y otra } \\
\text { para negocios de gran tamaño. } \\
\text { En los demás casos, el área comercial está consti- } \\
\text { tuida por empleados de la compańía. }\end{array}$ \\
\hline
\end{tabular}

\begin{tabular}{|l|l|}
\hline \multicolumn{1}{|c|}{ Criterio } & \multicolumn{1}{c|}{ Descripción } \\
$\begin{array}{l}\text { Número de } \\
\text { personas } \\
\text { en el área } \\
\text { comercial }\end{array}$ & $\begin{array}{l}\text { La empresa que es netamente comercial tiene } \\
\text { 200 de sus } 300 \text { empleados en esta área y cuenta } \\
\text { con } 27 \text { tiendas en todo el país. Tiene dos líneas } \\
\text { de negocio con dos marcas y almacenes diferen- } \\
\text { tes, y un director comercial para cada línea: una } \\
\text { directora es miembro de la familia y el otro no es } \\
\text { miembro de la familia. }\end{array}$ \\
$\begin{array}{l}\text { En otro caso, } 70 \text { de los 249 empleados se encuen- } \\
\text { tran en el área comercial. En esta, hay siete direc- } \\
\text { tores comerciales, uno para cada región del país. } \\
\text { Las dos fábricas cuentan con siete y diez emplea- } \\
\text { dos en el área comercial. } \\
\text { Las otras empresas tienen hasta veinte empleados } \\
\text { en el área. }\end{array}$ \\
$\begin{array}{l}\text { El mercado de casi todas es la región del país } \\
\text { cercana a su sede (norte- Caribe). Además, una } \\
\text { vende en el sur del país; dos, a nivel nacional; y } \\
\text { una exporta el 40\% de su producción a países de } \\
\text { América y Europa. }\end{array}$ \\
\hline
\end{tabular}

\section{Análisis de resultados en Barranquilla}

En las entrevistas realizadas a directores comerciales (o su equivalente) en Barranquilla, Colombia, se identificaron algunas decisiones que se repiten: comprar un lote grande de producto, retirar a alguna persona, vender a un cliente con retraso en el pago o con un plazo de pago muy extendido. Se pueden agrupar en temas de ventas, compras, personal, publicidad y finanzas. En el rubro de ventas, se encuentra la mayor cantidad de decisiones.

En las decisiones relacionadas con ventas, las influencias más comunes se relacionaron con la posición de la empresa en el mercado. Tal fue el caso de una compañía que incursionó con un producto nuevo y otra que no quería vender porque el cliente pedía un precio demasiado bajo. En estas dos decisiones, otro punto de influencia fue el margen que se obtendría al tomar la decisión. En otras decisiones, la influencia de la necesidad de reforzar la posición en el mercado se manifestó como ambición y ansias de vender. Otras influencias 
en este grupo de decisiones son el volumen a vender, el precio ofrecido por los competidores, la confianza que genera el cliente —así sea en un contacto breve- y el elemento religioso: «saldrá bien por fe». Hubo un caso en que el director comercial estaba a cargo de la empresa, pues su padre y gerente estaba de vacaciones, por lo que la decisión llenó una necesidad de autoafirmación. En varias de las decisiones, entre uno de los elementos a considerar, estaban las posibles consecuencias en el futuro, como no propiciar guerra de precios; la posibilidad de que la siguiente vez el cliente querría un precio más bajo; el hecho de que, en un futuro, el cliente podría comprar otros productos más rentables; o la posibilidad de dar servicio posventa de forma más ágil.

En las decisiones de compras, los puntos más importantes fueron la diferenciación de la competencia; el precio; el volumen; $y$, en concordancia, la fuerte inversión. En uno de estos casos, además, se consideraron las características culturales del mercado; de hecho, se consultaron clientes antes de tomar la decisión. Por el lado de las decisiones relacionadas con personal — casi todas implicaban retirar a algún empleado-, las influencias fueron ejercidas directamente por elementos afines a recursos humanos, como las características de personalidad del empleado, el favorecimiento de la promoción interna y la disminución del tiempo de inducción. Otra influencia destacada es la reducción de costos de la empresa, y otras son los resultados en ventas y el número de clientes potenciales no atendidos. En decisiones relativas a publicidad, el punto en común fue evaluar a quién iba dirigido el evento o pieza. La única decisión relacionada con finanzas -que implicaba un cambio en la forma como se calculan los costos fijos-, fue tomada por el área de Finanzas con la participación de la dirección comercial. En esta, hubo influencias asociadas con el cliente: que el precio de mercado no fuera a afectar la competitividad y que el asumir los costos no impactase el precio para el cliente.

En cuanto a los cargos involucrados en la toma de decisión, se identificó que, en casi todos los casos, tuvo algún papel la gerencia general (cuando el cargo de director comercial y el de gerente eran ejercidos por dos personas diferentes). Tal fue el escenario tanto en decisiones sobrevenidas - tomadas por otro o por influencia de alguien más, pero que afectaba o se ejecutaba en la dirección comercial- como en las tomadas directamente en la dirección comercial. No obstante, en las decisiones sobrevenidas, tuvo mucho mayor peso.

El papel de la gerencia fue de refuerzo en varios casos, aunque hubo una situación en que se intentó modificar la decisión. Sin embargo, en este caso, pesó más el rol de padre que el de gerente, pues la decisión se relacionaba con su temor por la seguridad de su hijo. También, hubo una decisión en que el gerente general y dueño se opuso a una decisión, pero los otros tres involucrados (vicepresidente de operaciones, director de la cuenta y gerente comercial) estaban a favor y primó su opinión por mayoría de votos. Hubo otros escenarios en que el gerente ralentizó. Hubo un par de casos en que un director de otra área o de otra unidad de negocio operó como elemento de objetividad o neutralidad, y, por eso, se le pidió su opinión En uno de ellos, por ejemplo, la persona no pertenecía a la familia, mientras que el gerente y la directora comercial son padre e hija.

Los subordinados fueron otro subsistema consultado en muchos casos. A veces, fueron quienes iniciaron el proceso de toma de decisión, como una compra a un nuevo proveedor, porque los vendedores notaron que el producto en otras presentaciones podría venderse más. 
Casi todos los subordinados reforzaron la decisión, menos aquel que quería vender a un cliente que exigía un precio demasiado bajo. Finalmente, el director comercial decidió no venderle.

Otras personas que influyeron en decisiones del área comercial pertenecían a las áreas financiera, almacén, compras, producción, entre otras. El papel de la persona de Finanzas fue directo en temas de alta inversión o de cartera, mientras que, en otros, desempeńó un rol de consultoría. Asumió un papel indirecto en una decisión tomada por la gerencia de participar en una feria, decisión que el director comercial ejecutó. Por lo general, cuando fue necesaria la participación de gerencia de planta o producción, se conformó como una influencia directa. En dos empresas, hay varios directores comerciales y los directores comerciales — colegas del entrevistado - estuvieron involucrados en una decisión: en un caso, como consultor y, en el otro, tuvo una influencia directa e incluso trató de parar la decisión, pero al final cedió. De las decisiones analizadas, esta fue la única que no resultó como se esperaba y la medida tuvo que ser anulada. Igualmente, varias decisiones procedieron por solicitud de un cliente, aunque a veces simplemente fue inspiración, pero no tuvo participación como tal en la decisión. Así, otro subsistema involucrado es el entorno: clientes y proveedores.

En las decisiones tomadas directamente por el director comercial, suelen intervenir más personas que en las sobrevenidas. Para las directas, hubo hasta seis entidades involucradas y solo una fue tomada nada más por el encargado del área comercial. Algunas de las decisiones sobrevenidas fueron tomadas únicamente por el gerente general, aunque hubo dos casos en que fueron cuatro los involucrados. En las decisiones sobrevenidas, el papel de la dirección comercial fue indirecto en más casos. A veces, sucedió que las decisiones sí estuvieron a cargo del director comercial, por ejemplo, las dos de publicidad: participar en feria y diseño de catálogo. Ambas fueron tomadas por la gerencia, pero el director comercial debía decidir cómo llevar a cabo la acción, el diseño.

En la Figura 4, se aprecia que, en la decisión, se involucraron seis subsistemas: el entorno, la empresa, información, otro personal de la empresa, subordinados y el director comercial. Las influencias entre subsistemas son cinco: cuatro son relativas y una fuerte. En cuanto a las fuentes de influencia, una la inicia y la refuerza, tres la promueven, y dos la refuerzan.

Figura 4. Representación de la decisión compra de un lote de productos

\section{E44 - D93 - Compra de un lote de productos muy grande}

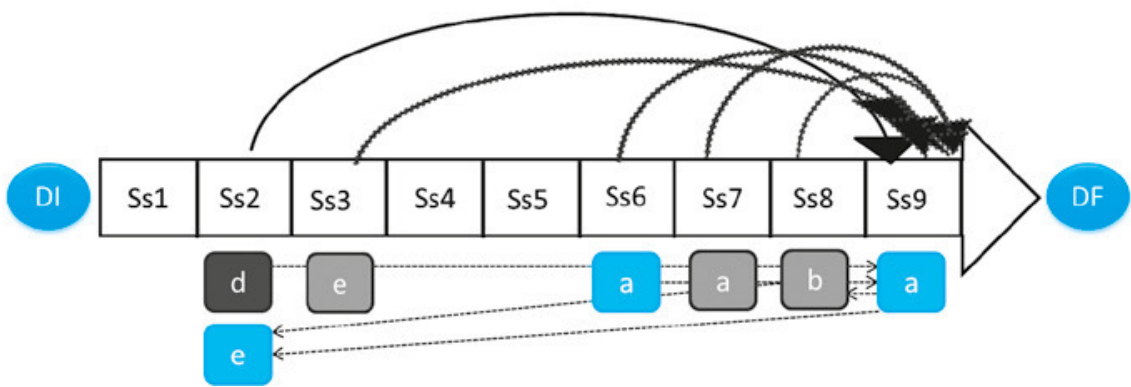


El entorno es el que inicia, pues la decisión surgió a partir de la propuesta de un proveedor. Al tratarse de una fuerte inversión, había temor de que los productos no se vendieran, pero, para la directora comercial, era atractivo el riesgo. Además de tomar la decisión, la empresa se diferenciaría de la competencia. En este caso, la dirección comercial, además de ventas, maneja compras.

\section{Comparación resultados de Cataluña y Barranquilla}

En ambas investigaciones, se encontró que los organismos de dirección - especialmente, el gerente general-y el entorno desempeñan un papel crucial en las decisiones del director comercial. En Cataluña, se identificó mayor acción del subsistema familia propiedad; en Barranquilla, fue fuerte siempre y cuando fuera, a su vez, parte de los órganos de dirección. Otro punto en común es que la mayoría de influencias promovieron la decisión. La diferencia se observa en que, en varias ocasiones, el director comercial en Cataluña tuvo un rol de ralentizar o modificar. Esta situación no se dio en Barranquilla, donde, más bien, el director comercial inició o promovió. En las pocas ocasiones en Barranquilla en las que alguien ralentizó, intentó modificar o parar, fue el gerente general u otro directivo no comercial. En ese sentido, en Cataluña, se encontraron fuerzas anuladoras, las que estuvieron ausentes en Barranquilla.

En ambos lugares, los asuntos relacionados con ferias, despido de personal, ubicación de un nuevo proveedor y lanzamiento de un nuevo producto se dieron como parte de las decisiones del director comercial. En Cataluña, se tomaron dos decisiones: establecer descuentos adicionales en determinadas ventas y dejar un cliente. Esta situación también se dio en Barranquilla, pero, en un caso, se conjugaron los dos temas en una sola decisión; es decir, se le dejó de vender a un cliente por solicitud del descuento adicional. Por otro lado, en Cataluña, se decidió contratar a una persona del área comercial despedida años atrás; en Barranquilla, ocurrió una situación similar, pero no había sido despedida de la empresa en cuestión, sino de una competidora ubicada en el mismo centro comercial. También, en ambas ciudades, se entrevistaron directores comerciales que, además de encargarse de las ventas, se hacen cargo de las compras. En este marco, se observa una diferencia: en Barranquilla, hubo más decisiones acerca de comprar lotes de productos para luego vender, mientras que en Cataluña los temas se enfocaron en compras de maquinaria o terrenos.

A partir de la comparación entre estos casos, se demuestra que, si bien la toma de decisiones utiliza la información como uno de sus elementos, se ve influida por diferentes fuentes que tanto en España como Colombia presentan claras similitudes. El estudio de Barranquilla (Colombia) confirma que la toma de decisión entre los directores comerciales es dinámica y compleja.

\section{Comparación resultados de Barranquilla en dos épocas}

En el estudio de Navia Núñez sobre la toma de decisiones estratégicas por parte de gerentes generales de Barranquilla, se identificó el siguiente proceso: «definición del problema, búsqueda de alternativas, evaluación de alternativas, selección de una alternativa (si había más de una), determinación de decisiones conexas a la alternativa escogida, e implementación de decisiones» (2002b, p. 148). En la investigación actual, a grandes rasgos, el proceso es similar, pues también se definió la situación, se buscaron alternativas, se evaluaron y se tomó la decisión. Las diferencias radican en que, en la actualidad, se les 
dio menos importancia a las decisiones conexas a la alternativa, se evaluaron menos alternativas y la evaluación de alternativas fue menos técnica. De hecho, ninguno enfatizó haber usado un esquema como diagramas y proyecciones — por escenarios, por ejemplo-, que fue una estrategia que se empleó en varios de los casos investigados en el año 2002. Además, aunque las empresas de ambos períodos acudieron a elementos como un análisis histórico de ventas, aquellas del período más reciente buscaron menos información interna y externa. Aun cuando los participantes del estudio anterior hayan usado más métodos técnicos para evaluar las alternativas, en aquel entonces, una de las conclusiones fue que las decisiones se tomaron pensando en el ingreso que pudieran representar y que el método no resultaba tan importante con tal de que diera un panorama más o menos claro de cuánto dinero puede entrar; si se requiere invertir; o, como mínimo, tratar de asegurarse de que vayan a entrar más recursos de los que pueden salir, a pesar de que no se sepa cuánto dinero es. Por último, un punto en común en ambos estudios es que las instituciones consultan a otras personas, lo que permite reducir la incertidumbre en la toma de decisión. Como consecuencia, aparecen agentes que influyen en dicho proceso.

\section{Discusión de resultados}

Stefanu señala que el análisis de las decisiones tomadas por los directores comerciales en empresas catalanas muestra

Individuos que sí son racionales y que actúan movidos por unas intenciones basadas en sus creencias y preferencias, pero que no evalúan alternativas, sino que toman una decisión, que les parece adecuada sin analizar otras posibles vías y que tampoco aplican el concepto de utilidad marginal ya que más bien, toman sus decisiones mediante mecanismos de razonamiento de carácter cualitativo tal como los describen Álvarez, Teira y Zamora (2005). (citado en 2011, p. 188)

De acuerdo con un hallazgo de Stefanu,

La decisión no es algo que nazca y muera en un único decisor, ni siquiera en un conjunto de decisores implicados en la toma de la decisión. En el proceso de la toma de decisiones intervienen agentes, tanto activos como pasivos, que de forma directa e indirecta impactan en la decisión, en la forma que tomará esta definitivamente o incluso en el proceso que se va a seguir para tomarla. Lo destacado de estos agentes es que no están implicados en la toma de decisión y que no les corresponde a ellos tomarla, es más, en algunos casos nos encontramos con agentes que son totalmente ajenos a la decisión que se va a tomar, puede que ni siquiera sean conscientes de que haya una decisión en proceso de ser tomada, pero no por ello dejan de influir en la misma ya sea de forma directa y clara o de forma sutil. La teoría de juegos que se aplica en los procesos de toma de decisiones nos acerca en parte a estos conceptos, pero se limitan a tratar situaciones en las que los implicados «juegan todos al mismo juego" pero no tiene en cuenta la influencia de aquellos otros agentes que «no están jugando en la partida». (2011, p. 189)

Al comparar los resultados obtenidos por Stefanu (2011) con la teoría del equilibrio de Nash (citado en Villar 2005), se nota que hay un claro aporte al describir que no todos los individuos que influyen en una decisión forman parte de esta, y que no todos son conscientes y partícipes de la misma.

Al analizar la contribución de los resultados de la investigación en Cataluña con respecto a la teoría de sistemas, se concluyó que lo hallado

Nos acerca al concepto que la teoría de sistemas promueve cuando habla del «estado de naturaleza», como aquellos elementos que influyen en los resultados de la 
decisión y sobre los que el decisor no controla. Aplicando el modelo de la teoría de sistemas, hemos podido descubrir que los «estados de naturaleza» no solo influyen en los resultados de la decisión, sino que influyen en el proceso de la toma de decisión moldeándola, frenándola, impulsándola e incluso anulándola y por lo tanto ejerciendo su influencia antes, incluso, de que la decisión haya sido tomada. (Stefanu, 2011, p. 190)

Además, Stefanu (2011) halló que los diagramas de flujo establecidos a partir de las entrevistas con los directores catalanes se asemejan a lo expresado por Mateos «En muchas ocasiones las decisiones son el resultado de interacciones, negociaciones y acciones políticas» (2007, p. 454). El estudio, también, encontró más grupos de influencia (nueve subsistemas, entre los cuales están los subordinados y la familia/propiedad) que los mencionados por otros autores. Por un lado, Valls (2003), Senlle (2002), Ariño (2005), León (1996) o Mateos (2007) reconocen la presencia de tres: decisor, empresa y grado de incertidumbre. Por otro, en el modelo de los tres círculos de Davis y Tagiuri, los grupos son familia, trabajo y propiedad (Trevinyo Rodríguez, citado en González, Castruita Morán y Mendoza, 2018).

Como resultado del análisis de las decisiones tomadas por los directores comerciales en las empresas familiares catalanas, a partir de la teoría de sistemas, se definió que la toma de decisiones se puede iniciar por la gestión de decisiones inherentes a su cargo, pero que fueron tomadas por otro; por decisión del director comercial fundamentada en situaciones de mercado o datos financieros; o por una decisión que afecta a varios departamentos, lo cual ocurre cuando el director comercial es el mismo propietario. El director comercial, más que analizar las repercusiones de las alternativas, pasa mucho tiempo gestionando por medio de relaciones interpersonales con personas que, a su vez, realizan una acción similar en su trabajo del día a día. Sucede, a veces, que el director comercial no busca nutrirse del entorno, sino que hay una dinámica en que el entorno mismo se hace sentir en el proceso de toma de decisión.

Al comparar los resultados con otros estudios del tema, se observa, por ejemplo, el realizado en Cali (Colombia) sobre una familia empresaria. Correa-Young, Restrepo y Acevedo indican que algunos miembros de la familia entrevistados consideraron

que no eran tenidos en cuenta para comunicarles cambios, proyectos y decisiones. No eran claras las funciones ni las líneas de reporte, lo que afectó la toma de decisión [...]. No había un subsistema ejecutivo que organizara y tuviera poder sobre los integrantes del grupo familiar. (2007, p. 90)

Los autores encontraron tres sistemas interactuantes complejos: la familia; la empresa familiar; y otros que incluyen el mercado, las políticas, las estrategias del negocio, los procesos operativos y de estructura y los aspectos financieros. «En estas interacciones confluían además diferentes experiencias, visión y energía para el manejo de problemas, toma de decisiones y capacidad para enfrentar los retos que imponía el ciclo de vida de la familia y el negocio" (Correa-Young et al., 2007, p. 92). Este estudio también utilizó una mirada sistémica, y llegó a establecer varios subsistemas e interacciones como sucede con la investigación del presente artículo.

En Quintana Roo (México), Esparza, García y Duréndez (2010) realizaron un estudio comparado entre empresas familiares y no familiares, mediante el cual hallaron que «los gerentes de empresas familiares utilizan en menor medida la información contable y financiera para la adecuada toma de decisiones, en contraste con los gerentes de las no familiares» (p. 42). 
Aunque el presente estudio no realizó la comparación con empresas no familiares, a través de la comparación del caso de Barranquilla con una investigación anterior - que abarcó tanto a empresas familiares como no familiares_- sí se encontró un menor uso de estas herramientas.

También en Quintana Roo, Navarrete y Sansores (2017) identificaron, por medio de una encuesta, que en las micro, pequeñas y medianas empresas la toma de decisiones es centralizada, informal e intuitiva. Los autores hallaron que el propietario tiende a participar en todas las decisiones de importancia, que hay información limitada y que el entorno influye en las decisiones. Así, coincide con los resultados de la presente investigación sobre la influencia del propietario y del entorno. Cabe anotar que Otero y Taddei (2018) determinaron que los intereses de la familia pueden tener mayor influencia que las condiciones del mercado.

Del mismo modo, Bracamonte y Figueredo Álvarez (2017) notaron cercanía del propietario con los procesos de producción o de comercialización en un estudio realizado entre auditores en Venezuela. Barrios (2015) llevó a cabo un análisis de caso en Argentina, en el que encontró que los roles de propietarios y los gerenciales se superponen. Dicha superposición puede suceder en empresas de diferentes tamaños, lo cual se asemeja a los resultados de la presente investigación, en los que se observa que el propietario se involucra en las decisiones, especialmente, si el director comercial no es miembro de la familia.

En otro orden de ideas, un tema importante en la modernidad es el uso de la tecnología y cómo puede influir en la toma decisiones. Por ejemplo, cuando los entrevistados del presente estudio indican que el propietario cambia constantemente lo ya planeado, refieren tanto al trato personal como también a distancia. Dado que muchos tienen un teléfono móvil o un computador a la mano, se apoyan en medios tecnológicos, como las redes sociales o el correo electrónico, incluso cuando viajan. Así, hay mayor inmediatez y menos restricciones para su influencia.

Con respecto a la tecnología, es importante mencionar el estudio de Medina y Carvajalino (2014, p. 126), que se enfoca en la influencia de sistemas de soporte como SAD en la toma de decisiones de dos empresas de Cartagena, Colombia. Los autores concluyeron que «diferentes sistemas de procesamiento de transacciones nutren desde las decisiones operativas estructuras; los sistemas de soporte y de apoyo a las decisiones que se generan en el área de gestión y estratégica funcionan de manera interrelacionada y en algunos casos de manera colaborativa» con otros módulos o áreas de la empresa (Medina y Carvajalino, 2014, p. 126). De este modo, se dieron cuenta de que este tipo de tecnologías robustas permiten que las decisiones sean más programadas. A partir de la investigación de Medina y Carvajalino (2014) y el presente estudio, se puede afirmar que los sistemas de soporte como SAD contribuyen a las decisiones planeadas, mientras que los servicios de mensajería instantánea pueden generar que decisiones ya tomadas se puedan cambiar rápidamente. En esa línea, en un análisis de empresas argentinas para el que realizó encuestas, Tovar (2017) deduce que el gerente debe analizar las herramientas disponibles — como bases de datos, redes sociales y tecnologías de la informaciónpara realizar un diagnóstico de la situación actual en la toma de decisiones, lo cual corrobora la influencia de estos elementos. De este modo, se visibiliza que, como indica Castells (2006), las redes sociales han cambiado las formas de comunicación y poder (citado en Torres, 2017).

Contabilidad y Negocios (14) 27, 2019 / ISSN 1992-1896 


\section{Conclusiones}

En el estudio enfocado en el caso de Cataluña, Stefanu concluyó que la decisión del director comercial no puede ser entendida si es analizada de forma aislada. Es necesario aplicar el nuevo paradigma que rige la teoría de sistemas y "contemplar la decisión como un todo integrado, no como un conjunto de partes» (Stefanu, 2011, p. 197). La autora señala que la toma de decisiones responde a un sistema complejo, debido a que está formado por varios subsistemas que interactúan. Existe un efecto combinado de los elementos que influyen en la decisión.

«Una decisión puede presentarse en forma de sistemas y subsistemas interrelacionados entre sí de forma dinámica», que ejercen influencia unos sobre otros: dan forma, moldean, modifican, paran o anulan una decisión durante el proceso (Stefanu, 2011, p. 198). En ese sentido, la decisión no nace y muere en un único decisor, «ni siquiera en un conjunto de decisores implicados en la toma de la decisión. En el proceso de la toma de decisiones, intervienen agentes, tanto activos como pasivos, que de forma directa e indirecta impactan en la decisión» (Stefanu, 2011, p. 189).

A partir del estudio comparado entre Cataluña y Barranquilla, se concluye lo siguiente:

- Los directores comerciales de Barranquilla evalúan más detenidamente las alternativas que los de Cataluña.

- Los directores comerciales en ambas localizaciones mostraron una fuerte interacción con diferentes subsistemas en la toma de decisiones.

- En las empresas familiares, hay un fuerte compromiso de la influencia de la familia, especialmente, si el gerente general es miembro de la familia, y el director no tiene una relación familiar con el gerente o propietario.

- En la mayor parte de los casos analizados, los miembros de la familia tienen mayor peso en la decisión que aquellos que no pertenecen a esta.

- El proceso de toma de decisiones por parte del director comercial en la empresa familiar catalana presenta particularidades que los otros modelos teóricos de toma de decisiones no han recogido hasta la fecha y que son similares a las particularidades de los directores comerciales de empresas familiares de Barranquilla. Ejemplo de ello es el caso del peso de la influencia de la estructura organizativa o la propia familia.

- Los diagramas creados por Stefanu (2011) pueden ser utilizados en otros contextos, tal y como se aplicó en Barranquilla, siempre y cuando se conozcan los subsistemas involucrados y los tipos de influencias. Los diagramas permiten realizar diagnósticos de la manera como se toman decisiones empresariales y pueden ser usados para entender cómo los subsistemas se afectan entre sí.

A partir de la comparación entre dos períodos distintos en Barranquilla, se establece que los directores comerciales suelen ser menos sistemáticos que los gerentes generales en la toma de decisión y tienden a tomar decisiones sobre la marcha, en lugar de seguir un proceso detallado.

Como posibles líneas para futuras investigaciones, se podrían establecer semejanzas y diferencias entre los elementos de influencia en la toma de decisiones en empresas no familiares. Asimismo, se sugiere realizar análisis comparativos con empresas de otros países, como Estados Unidos, Alemania o Perú. También, se recomienda considerar otros cargos gerenciales de la 
empresa, como el área financiera. Además, se puede profundizar en otras influencias sobre las decisiones, como la tecnología de la información.

\section{Referencias bibliográficas}

Aktouf, O. (1998). La administración: entre tradición y renovación. Cali: Universidad del Valle.

Álvarez Álvarez, J. F., Teira Serrano, D. y Zamora Bonilla, J. (2005). Filosofía de las ciencias sociales. Madrid: Universidad Nacional a Distancia.

Ariño, M. A. (2005). Toma de decisiones y gobierno de organizaciones. Barcelona: Deusto.

Artal, M. (2005). Dirección de ventas, organización del departamento de ventas y gestión de vendedores. Madrid: Esic.

Barrios, M. (2015). Superposición de roles gerenciales y roles propietarios en las pequeñas y medianas empresa familiares. Palermo Business Review, 12, 7-60. Recuperado de https://www.palermo.edu/economicas/cbrs/ pdf/pbr12/BusinessReview12_01.pdf

Bracamonte, O. y Figueredo Álvarez, C. (2017). La ética profesional, elemento contextualizante en la actuación del auditor en el sector público. Gestión y Gerencia, 11(2), 99-116. Recuperado de

Cachanosky, J. C. (2000). Las decisiones empresariales y las predicciones en economía. Revista Eseade, 32, 1-23. Recuperado de

Chiavenato, I. (2004). Teoría general de la administración. México: Mc Graw Hill.

Correa Young, A., Restrepo de Giraldo, L. y Acevedo, M. E. (2007). Más allá del protocolo de familia: un abordaje sistémico con la familia empresaria. Revista Pensamiento Psicológico, 3(9), 79-100. Recuperado de

Esparza A., J. L., García-Pérez de Lema, D., Duréndez Gómez-Guillamón, A. (2010). Diferencias de gestión financiera entre empresas familiares y no familiares del sector turístico mexicano. Actualidad Contable FACES, 13, (20), 29-48. Recuperado de
González; L. M. Castruita Morán, E. Mendoza, R. (2018). Caracterización de las empresas familiares: caso Durango (México). Pensamiento y Gestión 44, 103132. DOI: dx.doi.org/10.14482/pege.44.9617

http://portales.puj.edu.co/psicorevista/components/com_ joomlib/ebooks/R9_Pag_79-100.pdf

http://www.eseade.edu.ar/files/riim/Libertas/12_9_Cachanosky.pdf

http://www.saber.ula.ve/bitstream/123456789/32562/1/ articulo3.pdf

http://www.ucla.edu.ve/DAC/investigacion/gyg/GyG\%20 2017/Agosto\%202017/0-\%20GyG-\%20Mayo2017.pdf

Lazarsfeld, P. F., Berelson, B. y Gaudet, H. (1944). The People's Choice. New York: Duell Sloan and Pearce.

León, O. G. (1994). Análisis de decisiones, técnicas y situaciones aplicables a directivos y profesionales. Madrid: AC Graw Hill.

Mateos, P. (2007). Dirección y objetivos de la empresa actual. Madrid: Editorial Centro de Estudios Ramón Areces.

Medina Monterrosa, P. y Carvajalino Slaghekke, A. (2014). $\mathrm{Ni}$ al tigre ni al cuero: La toma de decisiones y el uso los sistemas de soporte y de ayuda a la decisión SSDSAD. Saber, Ciencia y Libertad 8(2), 115-127. https:// doi.org/10.18041/2382-3240/saber.2014v9n1.1994

Mintzberg, H. (1991). Mintzbergy la Dirección. Barcelona: Díaz de Santos.

Navarrete, J. E. y Sansores, E.A. (2017). La toma de decisiones en la micro, pequeña y mediana empresa mexicana: Un análisis de los factores determinantes. Gestión y Gerencia 11(2), 72-98.

Navia-Núńez, M. (2002a). Procesos de toma de decisiones estratégicas en empresas de Barranquilla. Revista Pensamiento y Gestión 13, 52-66.

Navia-Núñez, M. (2002b). Procesos de toma de decisiones en empresas de Barranquilla (tesis de maestría).

Contabilidad y Negocios (14) 27, 2019 / ISSN 1992-1896 
Universidad del Norte, División de Ciencias Administrativas, Barranquilla, Colombia.

Nuebauer, F. y Lank, Alden G. (1999). La empresa familiar, cómo dirigirla para que perdure. Bilbao: Ediciones Deusto, S.A.

Otero Flores, S. y Taddei Bringas, C. (2018). Competitividad de empresas familiares. Interciencia 43(4), 236-241. Recuperado de https://www.interciencia.net/wp-content/uploads/2018/05/236-6091-TADDEI-43-04.pdf

Prado Córdova, J., Alberton, A. y Lana, J. (2016). Un estudio sobre la clasificación de empresas hoteleras familiares y su desempeńo en las ciudades de Itapema y Porto Belo/SC/Brasil. Contabilidad y Negocios. Revista del Departamento Académico de Ciencias Administrativas 11(22), 35-51. https://doi.org/10.18800/ contabilidad.201602.003

Real Academia Española (2001). Diccionario de la lengua española. 22a ed. Ciudad de México: Espasa Calpe.

Sánchez-Crespo, A. J. y Sánchez, A. M. (2001). La empresa familiar: Manual para empresarios, claves legales para su correcta organización y su continuidad. Bilbao: Deusto.

Santesmases Mestres, M. (1999). Marketing, conceptos y estrategias. Madrid: Pirámide.

Senlle, A. (2002). Tomar decisiones y resolver problemas. Cómo potenciar las competencias del equipo. Barcelona: Gestión 2000.
Simon, H. (1972 [1947]). El comportamiento administrativo, estudio de los procesos decisorios en la organización administrativa. Madrid: Aguilar.

Stefanu-Lidorikiotu, Y. (2011). Elementos de la influencia en la toma de decisiones entre los directores comerciales de empresas familiares catalanas (tesis de doctorado). Ciencias del Trabajo, Universidad de Cádiz, Cádiz, España.

Torres, D. (2017). Representaciones sociales de docentes sobre la evaluación formativa mediadas por redes sociales. Íkala, Revista de Lenguaje y Cultura 22(2), 255-268. https://doi.org/10.17533/udea.ikala.v22n02a06

Tovar, C. (2017). Investigación sobre la aplicación de Business Intelligence en la gestión de las pymes de Argentina. Palermo Business Review 15, 79-97. Recuperado de https://www.palermo.edu/economicas/ cbrs/pdf/pbr15/PBR_15_05_Tovar.pdf

Valls, A. (2003). Las 12 habilidades directivas clave. Barcelona: Gestión 2000.

Villar, A. (2005). Decisiones sociales. Madrid: Mc Graw Hill.

Fecha de recepción: 16 de julio de 2018 Fecha de aceptación: 05 de diciembre de 2018 Correspondencia: contacto@infocaribe.info yanna@av-consultores.com antonio@av-consultores.com 